\title{
Hydroxysafflor yellow A exerts antioxidant effects in a rat model of traumatic brain injury
}

\author{
YANG WANG $^{1}$, CHUNHU ZHANG $^{1}$, WEIJUN PENG ${ }^{1}$, ZIAN XIA $^{1}$, \\ PINGPING GAN $^{1}$, WEI HUANG ${ }^{1}$, YAFEI SHI ${ }^{2}$ and RONG FAN ${ }^{1}$ \\ ${ }^{1}$ Department of Integrated Traditional Chinese and Western Medicine, Xiangya Hospital, \\ Central South University, Changsha, Hunan $410008 ;{ }^{2}$ College of Basic Medicine, \\ Guangzhou University of Traditional Chinese Medicine, Guangzhou, Guangdong 510006, P.R. China
}

Received July 31, 2015; Accepted August 15, 2016

DOI: $10.3892 / \mathrm{mmr} .2016 .5720$

\begin{abstract}
Free radical-induced oxidative damage occurs rapidly and is of primary importance during the secondary pathophysiological cascades of traumatic brain injury (TBI). Hydroxysafflor yellow A (HSYA) is a constituent of the flower petals of Carthamus tinctorius (safflower) and may represent a potential therapeutic strategy to improve outcomes following TBI. The present study aimed to identify HSYA in the brain tissues of rats exposed to TBI to determine its absorption and to investigate the underlying effects of HSYA on antioxidant enzymes in the brain tissues of TBI rats. To determine the absorption of HSYA for the investigation of the underlying antioxidant effects of HSYA in TBI, the presence of HSYA in the brain tissues of the TBI rats was identified using an ultra performance liquid chromatography-tandem mass spectrometry method. Subsequently, the state of oxidative stress in the TBI rat model following the administration of HSYA was investigated by determining the levels of antioxidant enzymes, including superoxide dismutase (SOD), malondialdehyde (MDA) and catalase (CAT), and the ratio
\end{abstract}

Correspondence to: Dr Rong Fan, Department of Integrated Traditional Chinese and Western Medicine, Xiangya Hospital, Central South University, 87 Xiangya Road, Changsha, Hunan 410008, P.R. China

E-mail: fanrong819@163.com

Abbreviations: TBI, traumatic brain injury; HSYA, hydroxysafflor yellow A; SOD, superoxide dismutase; MDA, malondialdehyde; CAT, catalase; GSH, glutathione; GSSG, glutathione disulfide; ROS, reactive oxygen species; $\mathrm{BBB}$, blood-brain barrier; UPLC-MS/MS, ultra performance liquid chromatography-tandem mass spectrometry; SD, Sprague-Dawley; CCI, controlled cortical impact; ESI- electrospray ionization source operated in negative mode; MRM, multiple reaction monitoring mode; TBA, thiobarbituric acid; ANOVA, one-way analysis of variance

Key words: hydroxysafflor yellow A, traumatic brain injury, oxidative stress, neuroprotective effect, ultra performance liquid chromatography-tandem mass spectrometry of glutathione (GSH)/glutathione disulfide (GSSG). The data obtained demonstrated that HSYA was absorbed in the brain tissues of the TBI rats. HSYA increased the activities of SOD and CAT, the level of GSH and the GSH/GSSG ratio. However, HSYA concomitantly decreased the levels of MDA and GSSG. These preliminary data suggest that HSYA has the potential to be utilized as a neuroprotective drug in cases of TBI.

\section{Introduction}

Traumatic brain injury (TBI) is a leading contributor to rates of mortality and permanent disability in individuals aged $<45$ years $(1,2)$. Almost 2,000,000 individuals sustain a TBI annually in the USA, contributing to one third of all cases of injury-associated mortality (3). In China, TBI accounts for between 38.7 and $57.3 \%$ of cases of road traffic accident-associated mortality (4). The costs of the long-term treatment and rehabilitation following TBI constitute a considerable burden on society (5), and its therapeutic efficacy remains unsatisfactory as its pathogenesis is driven by complex and interactive mechanisms (6).

Following TBI, secondary brain injury is the leading cause of TBI-associated mortality in hospital inpatients (7). Free radical-induced oxidative damage occurs rapidly and and is of primary importance during secondary pathophysiological cascades. During secondary brain injury in TBI, the brain is vulnerable to oxidative stress due to the high rate of oxidative metabolic activities, the abundance of polyunsaturated fatty acids and the relatively low levels of antioxidant enzyme activity (8). Morphological responses and neurobehavioral deficits deteriorate under the effects of oxidative neurodegeneration (9). The dynamic equilibrium between oxidants and antioxidants is disrupted following cerebral injury by the excessive consumption of antioxidants or accumulation of reactive oxygen species (ROS), or the two in combination (10). When the injured areas produce excess ROS following brain injury, superoxide $\left(\mathrm{O}_{2} \cdot\right)$ and nitric oxide $(\cdot \mathrm{NO})$ radicals are produced first, which become more potent oxidants through a series of reactions and metabolism, including peroxynitrite (ONOO-), hydroxyl $(\cdot \mathrm{OH})$, carbonate $\left(\mathrm{CO}_{3} \cdot\right)$ and nitrogen dioxide $\left(\cdot \mathrm{NO}_{2}\right)$ radicals (11). These byproducts further oxidize proteins, lipids, sugars and nucleotides (12-14). The above data demonstrate 
a significant early contribution of oxidative damage in the secondary injury response in TBI, reinforcing the requirement of improved antioxidant therapies.

Hydroxysafflor yellow A (HSYA; Fig. 1), a flavonoid compound extracted from Carthamus tinctorius L. (Asteraceae), has been used as an active marker compound for controlling the quality of safflower in the Chinese Pharmacopoeia (15). Previous studies have indicated that HSYA has cerebral protective effects (16) by reducing protein oxidation/nitration and lipid peroxides $(12,13)$, suppressing inflammatory responses (17) and attenuating breakdown of the blood-brain barrier (BBB) (12). Previous studies have also reported that HSYA may offer potential as a therapeutic strategy to improve outcomes following TBI $(18,19)$. However, no previous investigations have focused on the mechanism underlying the antioxidant activities of HSYA in a rat model of TBI. Thus, the present study aimed to determine the antioxidant effects of HSYA on TBI in rats.

In the present study, to determine the absorption of HSYA for investigation of the underlying antioxidant effects of HSYA in TBI, HSYA was identified in the brain tissues of TBI-induced rats using an ultra performance liquid chromatography-tandem mass spectrometry (UPLC-MS/MS) method. Subsequently, the state of oxidative stress in the TBI rat model following the administration of HSYA was estimated by determining the levels of superoxide dismutase (SOD), malondialdehyde (MDA) and catalase (CAT), in addition to the ratio of glutathione (GSH)/glutathione disulfide (GSSG).

\section{Materials and methods}

Plant materials and chemicals. HSYA (purity >98\%) was purchased from the National Institute for the Control of Pharmaceutical and Biological Products (Beijing, China). Gradient grade methanol for liquid chromatography was supplied by Merck Millipore (Darmstadt, Germany). Formic acid was obtained from Sinopharm Chemical Reagent Company (Shanghai, China) and high purity water was obtained from Wahaha Co., Ltd. (Hangzhou, China). The assay kits for SOD, MDA, CAT, GSH and GSSG, and Bradford protein were obtained from Nanjing Jiancheng Bioengineering Institute (Nanjing, China). All other reagents were of analytical grade.

Animals and surgicalprocedure.Healthy male Sprague-Dawley (SD) rats (weighing between 200 and $250 \mathrm{~g}$, age, 8-10 weeks) were supplied by the Laboratory Animal Research Center of Central South University (Changsha, China). The rats were housed in an environmentally controlled breeding room (22-25 $\mathrm{C}$; 12 -h light/dark cycle; $50 \pm 10 \%$ humidity) with access to a normal standard chow diet and tap water ad libitum. The animals were maintained under these conditions for at least 1 week, following which they were fasted for $12 \mathrm{~h}$ with free access to water prior to each experiment. All animal experiments were approved by the Central South University Animal Ethics Committee and conformed to the Guidelines for the Care and Use of Laboratory Animals.

The controlled cortical impact (CCI) model with TBI was established using an electronic controlled pneumatic impact device (TBI 0310; Precision Systems and Instrumentation

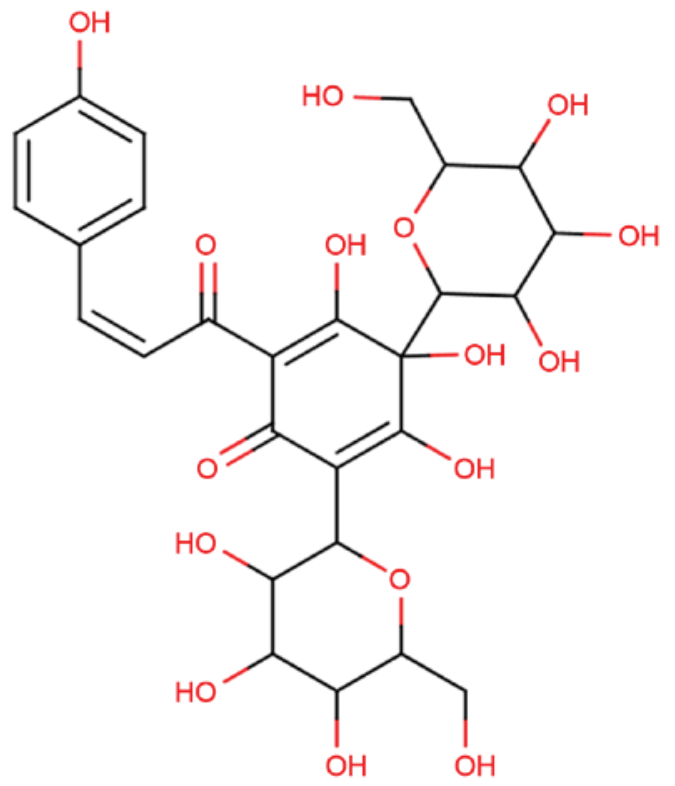

Figure 1. Chemical structure of hydroxysafflor yellow A.

LLC, Fairfax Station, VA, USA) under 3\% pentobarbital anesthesia $(50 \mathrm{mg} / \mathrm{kg})$, which was equipped with a hard stop Bimba cylinder (Bimba Manufacturing, Monee, IL, USA) and an impactor tip (external diameter, $5 \mathrm{~mm}$ ); this is an approved instrument for the TBI model. The parameters of the apparatus were as follows: Depth of impact, $5.00 \mathrm{~mm}$ from the cortical surface; impact velocity, $6.00 \mathrm{~m} / \mathrm{sec}$; dwell time, $500 \mathrm{msec}$. The body temperature of the rats was monitored throughout surgery, and a heated cage was used to maintain body temperature at $37.0 \pm 0.5^{\circ} \mathrm{C}$. Following surgery, the animals recovered fully in $\sim 20 \mathrm{~min}$, and the survival rate following surgery was $>90 \%$. A total of $96 \mathrm{SD}$ rats were randomly divided into the following four groups for the efficacy experiment: i) Vehicle control group, rats with TBI were intragastrically administered with $4 \mathrm{ml}$ normal saline vehicle $(0.9 \% \mathrm{NaCl})$; ii) sham operation group, rats underwent the same surgical procedures, but without trauma to the cerebral cortex; iii) $10 \mathrm{mg} / \mathrm{kg}$ HSYA treatment group, rats were orally administered with $10 \mathrm{mg} / \mathrm{kg}$ HSYA following trauma; iv) $30 \mathrm{mg} / \mathrm{kg}$ HSYA group, rats were orally administered with $30 \mathrm{mg} / \mathrm{kg}$ HSYA following trauma. Each group of rats were sacrificed by decapitation in an ice bath at 6, 12 and $24 \mathrm{~h}$ following intragastric administration. The ipsilateral cortex was immediately removed, placed in an ice bag and stored at $-80^{\circ} \mathrm{C}$ for biochemical assays, which were performed within 1 month.

Detection of HSYA in brain tissues using the UPLC-MS/MS) method. Following the oral administration of HSYA in rats subjected to TBI, the absorption of the compound in the brain tissue was determined by comparing the retention times and ion peaks with the authentic reference using the UPLC-MS/MS method.

The Acquity TQD UPLC-MS/MS system (Waters Corporation, Milford, MA, USA), consisting of Acquity UPLC online SPE manager (OSM), Acquity UPLC binary solvent manager, Acquity UPLC column manager, Acquity UPLC sample manager, Acquity TQ detector, masstrack 
online SPE cartridges and Acquity UPLC online SPE manager software (MassLynx) was used. Analyses were performed under an electrospray ionization source, which was operated in the negative mode (ESI-). A Waters Acquity UPLC BEH $\mathrm{C}_{8}$ $(2.1 \times 100 \mathrm{~mm} ; 1.7 \mu \mathrm{m})$ column with methanol (A) and $0.1 \%$ formic acid in water (B) was used at a flow rate of $0.3 \mathrm{ml} / \mathrm{min}$ to establish the elution gradient (A:B ratios: $0 \mathrm{~min}, 10: 90$; 5 min, 60:40; 6 min, 90:10; 7 min, 90:10; 8 min, 10:90). The column temperature was set at $30^{\circ} \mathrm{C}$. The injection volume was $5 \mu \mathrm{l}$ using full-loop mode. The detection wavelengths of the photodiode array detector were set at 200-600 nm. For MS/MS detection, the following parameters were used: Temperature of source gas (nitrogen), $110^{\circ} \mathrm{C}$; desolvation gas (nitrogen) flow, $650 \mathrm{l} / \mathrm{h}$ at $365^{\circ} \mathrm{C}$; capillary voltage, $2.5 \mathrm{KV}$; cone voltage, $26 \mathrm{~V}$; cone gas flow, $50 \mathrm{l} / \mathrm{h}$; collision gas (argon) flow, $0.2 \mathrm{ml} / \mathrm{min}$. Detection was performed in the multiple reaction monitoring mode (MRM).

The HSYA (30 mg/kg) was administered to the TBI rats, and the whole brain was rapidly removed following decapitation 30 min later. The surface blood products were cleared with ice-cold $\mathrm{ddH}_{2} \mathrm{O}$, and the samples were dissected and homogenized in $4 \mathrm{ml}$ ice-cold methanol using a TissueLyser LT homogenizer (Qiagen GmbH, Hilden, Germany). The homogenates were centrifuged at $1,500 \times g$ at $4^{\circ} \mathrm{C}$ for $10 \mathrm{~min}$, and the supernatant was collected separately for evaporation to dryness under nitrogen at $37^{\circ} \mathrm{C}$. Each dry extract was dissolved in $200 \mu 1$ methanol (20\%) and then centrifuged at 7,500 $\mathrm{x} g$ at $4^{\circ} \mathrm{C}$ for $15 \mathrm{~min}$. The upper layer was filtered through a $0.22 \mu \mathrm{m}$ nylon filter, and $5 \mu \mathrm{l}$ was injected automatically to UPLC-MS/MS for analysis.

Estimation of antioxidant and oxidative status. The stored cortices were weighed, dissected and homogenized with nine volumes $(1: 9, \mathrm{w} / \mathrm{v})$ of ice-cold normal saline in a homogenizer (TissueLyser LT; Qiagen, GmbH). The homogenates were centrifuged at $1,500 \times g$ at $4^{\circ} \mathrm{C}$ for $15 \mathrm{~min}$. The supernatants were used to measure the oxidative product contents, antioxidant enzyme activities and redox status, according to the manufacturer's protocols for the reagent kits (Nanjing Jiancheng Bioengineering Institute). Tissue protein concentrations were measured using the Bradford method (20).

The cortical levels of MDA were estimated using the thiobarbituric acid (TBA) method, as described by Zhao et al, with minor modifications (21). According to the manufacturer's protocol, the supernatant and TBA were mixed together and incubated at $95^{\circ} \mathrm{C}$ for $40 \mathrm{~min}$. The reaction mixture was cooled to room temperature under flowing water and centrifuged at $1,750 \mathrm{x} g$ for $10 \mathrm{~min}$ at $4^{\circ} \mathrm{C}$. The upper layer was used to determine the change in absorbance on a spectrophotometer at $532 \mathrm{~nm}$.

In measuring the activities of the antioxidant enzymes, the supernatant obtained was used to determine the activities of SOD and CAT. The detailed procedures were in accordance with the instructions of the assay kits supplied by Nanjing Jiancheng Bioengineering Institute. The absorbance of the test solution was measured at a wavelength of $450 \mathrm{~nm}$ for SOD activity and $405 \mathrm{~nm}$ for CAT activity. The activities of SOD and CAT were corrected with protein quantity and expressed as the fold change relative to the control, as $\mathrm{U} / \mathrm{mg}$ of protein.
The levels of GSH and GSSG in the cortices were determined to assess the redox status. The protocol, according to the specification of the GSH/GSSG assay kit (Nanjing Jiancheng Bioengineering Institute), was previously reported by Zhao et al (21). The absorbance was recorded at $405 \mathrm{~nm}$ on a spectrophotometer and then used to calculate the GSH/GSSG ratio.

Statistical analysis. All data were analyzed using SPSS 15.0 software (SPSS, Inc. Chicago, IL, USA). One-way analysis of variance and Dunnett's $t$-test were used to determine the significant differences between the four groups. All parameters are expressed as the mean \pm standard deviation. $\mathrm{P}<0.05$ was considered to indicate a statistically significant difference.

\section{Results}

Detection of HSYA using UPLC-MS/MS. The overall intraand inter-day variations were $<5 \%$ for HSYA, and the method was reproducible with good precision. The accuracy assessments were performed using recovery assessment, and the recovery of all compounds was $>90 \%$. These results suggested that the UPLC-MS/MS method was suitable for the detection of HSYA. As shown in Fig. 2, the predominant mass transitions of HSYA were $\mathrm{m} / \mathrm{z} 611.18 \rightarrow 491.04$, according to the UPLC-MS/MS method. The precursor ion was detected at $\mathrm{m} / \mathrm{z}$ $611[\mathrm{M}-\mathrm{H}]^{-}$. In the production mass spectra of $[\mathrm{M}-\mathrm{H}]^{-}, \mathrm{m} / \mathrm{z} 491$ was the prominent fragment ion. HSYA was identified in the extracts or biopsies based on its retention time $(2.74 \pm 0.02 \mathrm{~min})$, and characteristic $[\mathrm{M}-\mathrm{H}]^{-}$ion $(\mathrm{m} / \mathrm{z}$ 611.18) and fragment ions (m/z 491.04). Representative MRM chromatograms of the brain tissues from the untreated (drug-free), HYSA-treated and TBI+HYSA administration rats are shown in Fig. 3A-C, respectively.

Effects of antioxidant assay. As shown in Fig. 4, significantly higher levels of MDA were measured in the brains of the TBI (vehicle) rats, compared with the sham group $(\mathrm{P}<0.01)$. Treatment with HSYA (10 and $30 \mathrm{mg} / \mathrm{kg}$ ) significantly decreased the levels of MDA at different time points $(6,12$ and $24 \mathrm{~h}$ ), compared with the vehicle $(\mathrm{P}<0.05$ and $\mathrm{P}<0.01)$. The maximum alteration was observed at $24 \mathrm{~h}$.

Compared with the sham treatment group, cerebral injury in the TBI rats led to a significant decline in the activities of SOD and CAT, as shown in Fig. $5(\mathrm{P}<0.01)$. The administration of HSYA (10 and $30 \mathrm{mg} / \mathrm{kg}$ ) caused a significant increase in the levels of SOD at different time points $(6,12$ and $24 \mathrm{~h})$, compared with the vehicle-treated group $(\mathrm{P}<0.05$ and $\mathrm{P}<0.01)$, particularly following treatment with the high dose of HSYA $(30 \mathrm{mg} / \mathrm{kg}$ ). The activity of CAT increased significantly $12 \mathrm{~h}$ following treatment with the low dose of HSYA $(10 \mathrm{mg} / \mathrm{kg})$, compared with the vehicle treatment $(\mathrm{P}<0.05)$. Following treatment with a high dose of HSYA $(30 \mathrm{mg} / \mathrm{kg}$ ), the activities of CAT were significantly increased at all time points $(6,12$ and $2 \mathrm{~h}$ ), compared with the vehicle treatment $(\mathrm{P}<0.05$ and $\mathrm{P}<0.01)$.

As shown in Table I, brain injury reduced the levels of GSH and the GSH/GSSG ratios, and simultaneously increased levels of GSSG (all $\mathrm{P}<0.01$ ), compared with the sham group. The treatments with HSYA (10 and $30 \mathrm{mg} / \mathrm{kg}$ ) significantly increased the levels of GSH and GSH/GSSG ratios, and 


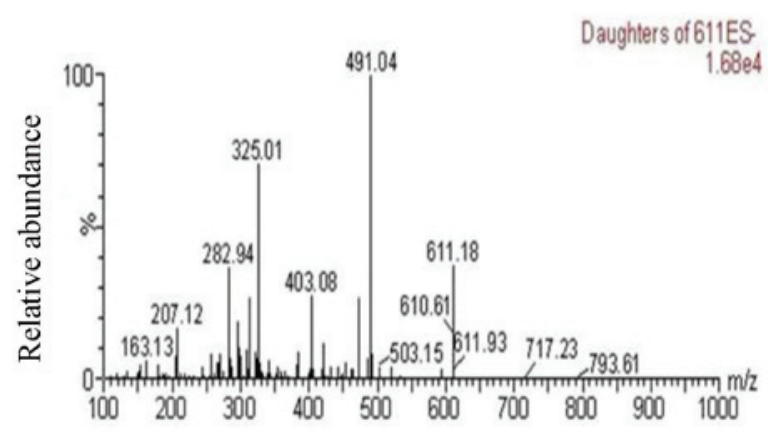

Figure 2. Mass spectrum of hydroxysafflor yellow A.

A

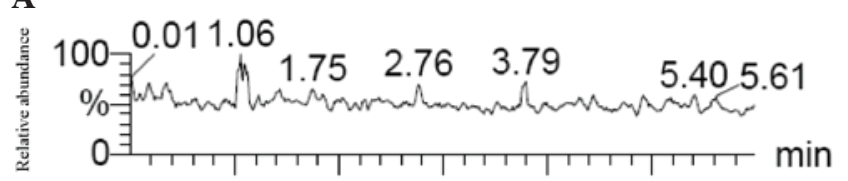

B

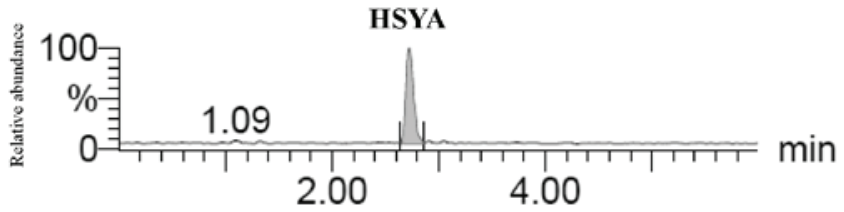

C

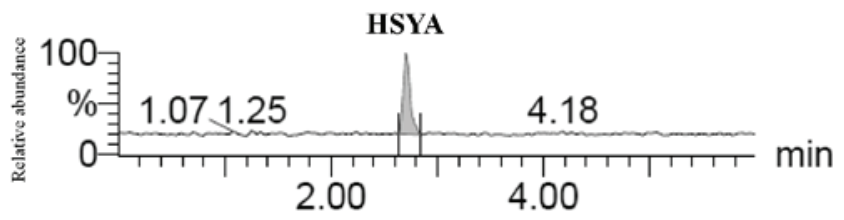

Figure 3. Detection of HSYA in brain tissues of TBI rats using ultra-high performance liquid chromatography with tandem mass spectrometry. Representative multiple reaction monitoring chromatograms of HSYA in (A) untreated brain tissue, (B) normal brain tissue treated with reference HSYA and (C) brain tissue post-TBI and 30 min following intragastric administration of HSYA $(30 \mathrm{mg} / \mathrm{kg})$. HYSA, hydroxysafflor yellow A; TBI traumatic brain injury.

decreased the levels of GSSG in the cerebral tissues of the TBI rats, compared with the vehicle group.

\section{Discussion}

The present study demonstrated that, according to the UPLC-MS/MS method, HSYA was absorbed in the brain tissues of TBI rats. Treatment of the TBI rats with HSYA significantly alleviated the imbalance between oxidants and antioxidants, resulting in further neuroprotective effects. HSYA increased the activities of SOD and CAT, the level of GSH and the GSH/GSSG ratio. In addition, HSYA concomitantly decreased the levels of MDA and GSSG. The above preliminary data provided evidence suggesting that HSYA offers potential to be utilized as a neuroprotective drug for TBI.

To establish a link between the absorbed chemical compound and its biological activity, HSYA was detected in

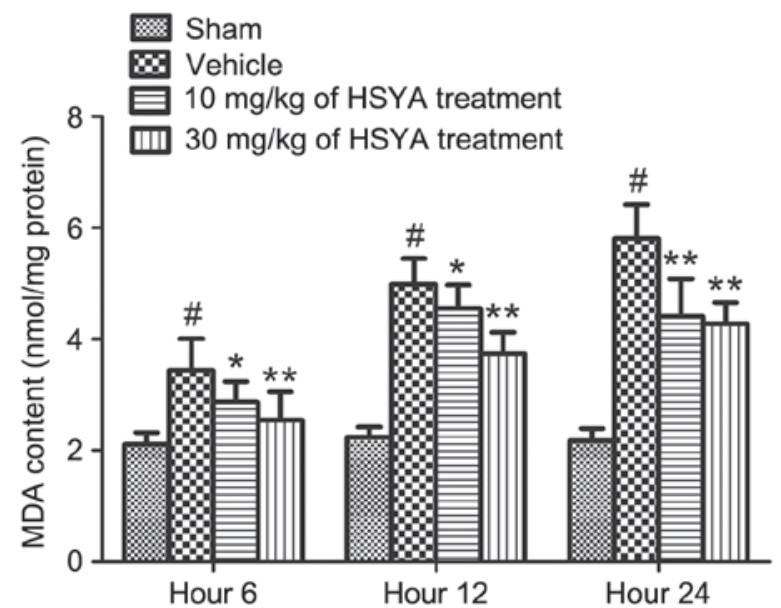

Figure 4. Effects of HSYA (10 and $30 \mathrm{mg} / \mathrm{kg}$ ) on the levels of MDA in rat brain tissues at different time points. Values are expressed as the mean \pm standard deviation. " $\mathrm{P}<0.01$, compared with the sham group at the same time point, ${ }^{*} \mathrm{P}<0.05$, compared with the vehicle group at the same time point, ${ }^{* *} \mathrm{P}<0.01$, compared with the vehicle group at the same time point. HYSA, hydroxysafflor yellow A; MDA, malondialdehyde.

the brain tissues of TBI rats using the UPLC-MS/MS method. If no evidence of absorption is found, any investigation of an effect-associated component is most likely to be incorrect (22). Thus, in the present study, a sensitive and accurate UPLC-MS/MS method was successfully developed to detect HSYA in the brain tissues of the TBI rats. The pretreatment of samples makes this method easy to perform in a short period of time, and the method of analysis conforms to the criteria for validation of the UPLC-MS/MS method including calibration curve, intra- and inter-day precisions and relative standard deviation of recovery (19). According to the mass spectrum of the HSYA, as shown in Fig. 2, two mass transitions, which followed were $611 \rightarrow 491.04 \mathrm{~m} / \mathrm{z}$ and $611 \rightarrow 325.06 \mathrm{~m} / \mathrm{z}$. These two MRM chromatograms were used to record the response. The HSYA in the biopsies were identified by comparing the retention time and ion peaks (Fig. 3). The results provided evidence that HSYA was absorbed in the brains of the TBI rats. The detection of this bioactive compound may assist in elucidating the direct pharmacological actions of HSYA for the treatment of TBI, and enables further investigation of the effects of HSYA on oxidative stress in the rat model of TBI.

HSYA is a hydrophilic drug with low oral bioavailability, belonging to the biopharmaceutics classification system III 


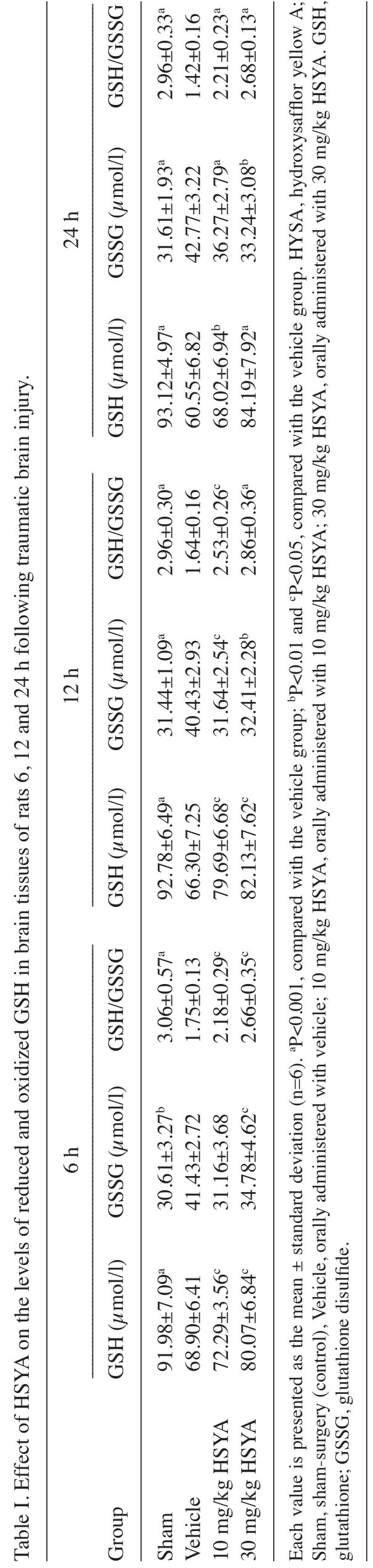
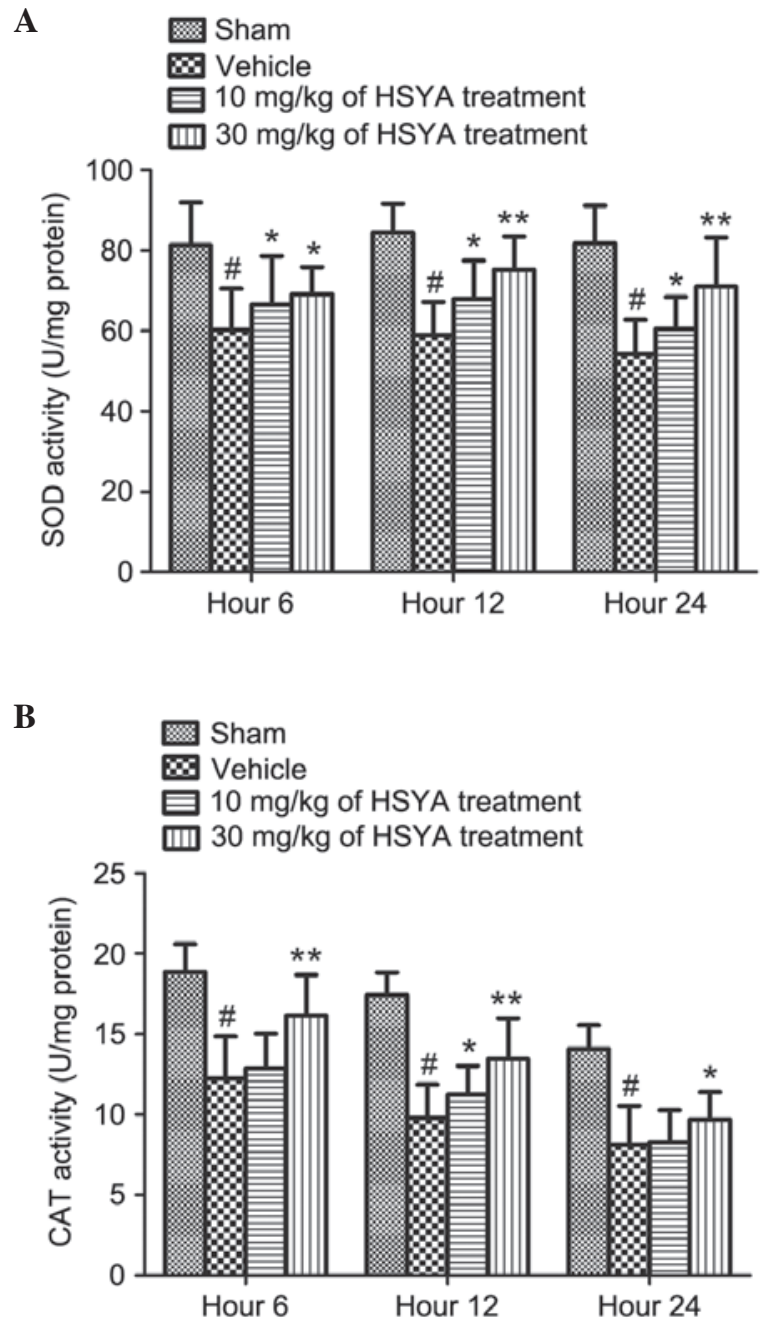

Figure 5. Effects of HSYA (10 and $30 \mathrm{mg} / \mathrm{kg}$ ) on the levels of SOD and CAT. The levels of (A) SOD and (B) CAT in the rat brain tissues were examined at different time points. Values are expressed as the mean \pm standard deviation. ${ }^{\#} \mathrm{P}<0.01$, compared with the sham group at the same time point; ${ }^{*} \mathrm{P}<0.05$, compared with the vehicle group at the same time point; ${ }^{* *} \mathrm{P}<0.01$, compared with the vehicle group at the same time point. HYSA, hydroxysafflor yellow A; SOD, superoxide dismutase; CAT, catalase.

class of drugs $(23,24)$. A previous study confirmed that HSYA can cross the BBB (19) and, consequently, HSYA has been administered via injection in several studies for investigating the treatment of cerebral diseases $(18,25,26)$. Of note, following TBI, small molecules (286-10,000 Da) are able to enter the brain up to a few days following injury due to BBB disruption (27). Therefore, HSYA, with a molecular weight of $611 \mathrm{Da}$, is more readily absorbed into the brains of TBI rats, compared with normal rat (28). In addition, the period of potential secondary damage from barrier disruption and the period during which HSYA has direct access to the injured brain may be longer than previously suggested (27). This leads to the accumulation of higher levels of HSYA in the brain tissues of TBI rats, which may have neuroprotective effects. Thus, the disruption of the BBB following TBI enables HSYA to cross the barrier and exert antioxidative effects.

Oxidative stress is important in the pathogenesis of secondary brain injury following TBI (29). Brain tissue is enriched with fatty acids, which are readily peroxidized, 
consumes a substantial proportion $(20 \%)$ of total oxygen consumption for its relatively low weight $(2 \%)$, and has limited antioxidant defenses (30). In addition, the cerebral metabolic rate is 7.5 -fold higher than the average metabolic rate (31). Due to its higher metabolic rate and lipid content, the brain is considered to be particularly sensitive to oxidative damage following TBI. TBI generally results in tardive neurological dysfunction and death through the processes of secondary injury $(32,33)$. It is associated with a mechanism involving excess excitatory amino acids (28), ionic imbalance (34), anomalous proteolytic enzyme activity (35), a low level of ATP (36) and oxidative stress. Among these, continued oxidative stress is a significant contributor to the deterioration of neurological function (37). Neurological function is vulnerable to injury by oxidative stress as its rate of oxygen consumption is high, nerve cells are not readily replenished, and the high levels of polyunsaturated fatty acids in the brain are targets of the lipid peroxidation initiated by ROS (38). The accumulated ROS include superoxide $\left(\mathrm{O}_{2}{ }^{*}\right)$, hydroxyl radical $(\cdot \mathrm{OH})$, hydrogen peroxide $\left(\mathrm{H}_{2} \mathrm{O}_{2}\right)$ and hypochlorous acid $(\mathrm{HOCl})$, which induces protein oxidation, inhibits the mitochondrial electron transport chain, cleaves DNA and leads to the peroxidation of cellular and vascular structures (39). The above processes lead to hypoperfusion, disruption of axonal guidance, disordered metabolism and brain edema (40).

MDA is the end product of membrane lipid peroxidation, which is generated from cellular membrane damage in oxidative stress (41). Therefore, it is widely considered to be a valuable index for measuring the extent of oxidative stress. SOD and CAT enzymes are crucial mediators in scavenging rapidly generated oxygen free radicals following TBI. SOD is a first line anti-oxidative enzyme, which is responsible for the defense mechanism against ROS and other superoxide anion-free radicals (42). SOD first catalyzes the transformation of superoxide radicals to $\mathrm{H}_{2} \mathrm{O}_{2}$ (43), following which CAT transforms $\mathrm{H}_{2} \mathrm{O}_{2}$ into $\mathrm{H}_{2} \mathrm{O}$ and $\mathrm{O}_{2}$ (44). The GSH system is well known to protect cells against oxidative damage in the pivotal role of redox homeostasis. It consists of reduced and oxidized forms of GSH. GSH is a predominant intracellular nonenzymatic antioxidant in tissues. During the processes of oxidative damage, GSH binds directly to oxygen free radicals to facilitate the conversion of $\mathrm{H}_{2} \mathrm{O}_{2}$, to then become GSSG $(45,46)$. The measurement of GSH/GSSG is a useful indicator of oxidative stress and can be used to monitor antioxidant effects (46).

Accordingly, the present study determined the levels of MDA, SOD and CAT, and the GSH/GSSG ratio to investigate the antioxidant effects. The results of the present study showed that HSYA treatment significantly prevented the increases in the levels of MDA, SOD and CAT. Furthermore, the levels of GSH were increased and the levels of GSSG were decreased following treatment with HSYA, compared with those in the vehicle group. The elevation of the GSH/GSSG ratio in the present study also reflects the anti-oxidative effect of HSYA. These results suggested that HSYA attenuated the excessive formation of free radicals secondary to TBI. Further investigations are required to investigate the detailed mechanisms of the signaling pathway underlying the effects of HSYA on brain cells against oxidative stress in vivo and in vitro.

In conclusion, treatment with the absorbed bioactive compound HSY attenuated oxidative injury to markedly inhibit neuronal injury following TBI, indicating that HSYA offers potential in TBI treatment as a neuroprotective therapy for the prevention of oxidative stress. The present study presented a canonical method to elucidate the mechanism of action in herbal chemicals, and suggested that HSYA may be a promising therapeutical compound for the treatment of TBI.

\section{Acknowledgements}

This study was supported by the National Natural Science Foundation of China (grant nos. 81303074, 81403259, 81202781 and 81303098) and the Administration of Traditional Chinese Medicine of Hunan Province, China (grant no. 201455).

\section{References}

1. Harrison-Felix C, Kolakowsky-Hayner SA, Hammond FM, Wang R, Englander J, Dams-O'Connor K, Kreider SE, Novack TA and Diaz-Arrastia R: Mortality after surviving traumatic brain injury: Risks based on age groups. J Head Trauma Rehabil 27: E45-E56, 2012.

2. Roozenbeek B, Maas AI and Menon DK: Changing patterns in the epidemiology of traumatic brain injury. Nat Rev Neurol 9: 231-236, 2013.

3. Corps KN, Roth TL and McGavern DB: Inflammation and neuroprotection in traumatic brain injury. JAMA Neurol 72: 355-362, 2015.

4. Wu X, Hu J, Zhuo L, Fu C, Hui G, Wang Y, Yang W, Teng L, Lu S and $\mathrm{Xu} \mathrm{G}$ : Epidemiology of traumatic brain injury in eastern China, 2004: A prospective large case study. J Trauma 64: 1313-1319, 2008.

5. Tuominen R, Joelsson P and Tenovuo O: Treatment costs and productivity losses caused by traumatic brain injuries. Brain Inj 26: 1697-1701, 2012.

6. Lei J, Gao G and Jiang J: Acute traumatic brain injury: Is current management evidence based? An empirical analysis of systematic reviews. J Neurotrauma 30: 529-537, 2013.

7. Kou K, Hou XY, Sun JD and Chu K: Current pre-hospital traumatic brain injury management in China. World J Emerg Med 5: 245-254, 2014

8. Jin W, Wang H, Yan W, Zhu L, Hu Z, Ding Y and Tang K: Role of Nrf2 in protection against traumatic brain injury in mice. J Neurotrauma 26: 131-139, 2009.

9. Smith JA, Park S, Krause JS and Banik NL: Oxidative stress, DNA damage and the telomeric complex as therapeutic targets in acute neurodegeneration. Neurochem Int 62: 764-775, 2013.

10. Adibhatla RM and Hatcher JF: Lipid oxidation and peroxidation in CNS health and disease: From molecular mechanisms to therapeutic opportunities. Antioxid Redox Sign 12: 125-169, 2010.

11. Hall ED, Detloff MR, Johnson K and Kupina NC: Peroxynitrite-mediated protein nitration and lipid peroxidation in a mouse model of traumatic brain injury. J Neurotrauma 21: 9-20, 2004.

12. Sun L, Yang L, Xu YW, Liang H, Han J, Zhao RJ and Cheng Y: Neuroprotection of hydroxysafflor yellow A in the transient focal ischemia: Inhibition of protein oxidation/nitration, 12/15-lipoxygenase and blood-brain barrier disruption. Brain Res 1473: 227-235, 2012.

13. Fan L, Dang X, Shi Z, Zhang C and Wang K: Hydroxysafflor yellow A protects PC12 cells against the apoptosis induced by oxygen and glucose deprivation. Cell Mol Neurobiol 31: 1187-1194, 2011.

14. Gracy RW, Talent JM, Kong Y and Conrad CC: Reactive oxygen species: The unavoidable environmental insult? Mutat Res 428: 17-22, 1999.

15. Pharmacopoeia Commission of PRC: Pharmacopoeia of the People's Republic of China, Chemical Industry Press, Beijing: 141, 2010.

16. Qi Z, Yan F, Shi W, Dong W, Zhao Y, Shen J, Ji X, Liu KJ and Luo Y: AKT-related autophagy contributes to the neuroprotective efficacy of hydroxysafflor yellow A against ischemic stroke in rats. Transl Stroke Res 5: 501-509, 2014.

17. Li J, Zhang S, Lu M, Chen Z, Chen C, Han L, Zhang M and Xu Y: Hydroxysafflor yellow A suppresses inflammatory responses of BV2 microglia after oxygen-glucose deprivation. Neurosci Lett 535: 51-56, 2013. 
18. Bie XD, Han J and Dai HB: Effects of hydroxysafflor yellow A on the experimental traumatic brain injury in rats. J Asian Nat Prod Res 12: 239-247, 2010.

19. Guo Y, Wang Y, Huang X, Lv H, Fan R, Huang W, Gan P, Liu W, Yan K, Xia Z and Liu J: Determination of hydroxysafflor yellow A in biological fluids of patients with traumatic brain injury by UPLC-ESI-MS/MS after injection of Xuebijing. Biomed Chromatogr 28: 1090-1095, 2014.

20. Sharma SK, Babitch JA: Application of Bradford's protein assay to chick brain subcellular fractions. J Biochem Biophys Methods 2: 247-250, 1980.

21. Zhao H, Chai W, Gao W, Xu L, Zhang H and Yang Y: Hyperoxygenated solution: Effects on acute hypobaric hypoxia-induced oxidative damage in rabbits. High Alt Med Biol 10: 283-291, 2009

22. Wang Y, Huang X, Liang Q, Fan R, Qin F, Guo Y, Yan KP, Liu W, Luo JK, Li YH, et al: A strategy for detecting absorbed bioactive compounds for quality control in water extract of rhubarb by ultra performance liquid chromatography with photodiode array detector. Chin J Integr Med 18: 690-698, 2012.

23. Qi J, Zhuang J, Wu W, Lu Y, Song Y, Zhang Z, Jia J and Ping Q: Enhanced effect and mechanism of water-in-oil microemulsion as an oral delivery system of hydroxysafflor yellow A. Int J Nanomedicine 6: 985-991, 2011.

24. Lv LZ, Tong CQ, Yu J, Han M and Gao JQ: Mechanism of enhanced oral absorption of hydrophilic drug incorporated in hydrophobic nanoparticles. Int J Nanomedicine 8: 2709-2717, 2013.

25. Wei X, Liu H, Sun X, Fu F, Zhang X, Wang J, An J and Ding H: Hydroxysafflor yellow A protects rat brains against ischemia-reperfusion injury by antioxidant action. Neurosci Lett 386: 58-62, 2005.

26. Zhu H, Wang Z, Ma C, Tian J, Fu F, Li C, Guo D, Roeder E and Liu K: Neuroprotective effects of hydroxysafflor yellow A: In vivo and in vitro studies. Planta Med 69: 429-433, 2003.

27. Habgood MD, Bye N, Dziegielewska KM, Ek CJ, Lane MA, Potter A, Morganti-Kossmann C and Saunders NR: Changes in blood-brain barrier permeability to large and small molecules following traumatic brain injury in mice. Eur J Neurosci 25 : 231-238, 2007.

28. Wang CY, Liu Q, Huang QX, Liu JT, He YH, Lu JJ and Bai XY: Activation of PPAR $\gamma$ is required for hydroxysafflor yellow A of Carthamus tinctorius to attenuate hepatic fibrosis induced by oxidative stress. Phytomedicine 20: 592-599, 2013.

29. Mojtahedzadeh M, Ahmadi A, Mahmoodpoor A, Beigmohammadi MT, Abdollahi M, Khazaeipour Z, Shaki F, Kuochaki B and Hendouei N: Hypertonic saline solution reduces the oxidative stress responses in traumatic brain injury patients. J Res Med Sci 19: 867-874, 2014.

30. Gan L, Wang ZH, Zhang H, Zhou R, Sun C, Liu Y, Si J, Liu YY and Wang ZG: Protective effects of shikonin on brain injury induced by carbon ion beam irradiation in mice. Biomed Environ Sci 28: 148-151, 2015

31. Li J and Wang Y: Effect of different methods of hypoxic exercise training on free radical oxidation and antioxidant enzyme activity in the rat brain. Biomed Rep 1: 925-929, 2013.
32. Xie Z, Lei B, Huang Q, Deng J, Wu M, Shen W and Cheng Y: Neuroprotective effect of Cyclosporin A on the development of early brain injury in a subarachnoid hemorrhage model: A pilot study. Brain Res 1472: 113-123, 2012.

33. Yakovlev AG, Knoblach SM, Fan L, Fox GB, Goodnight R and Faden AI: Activation of CPP32-like caspases contributes to neuronal apoptosis and neurological dysfunction after traumatic brain injury. J Neurosci 17: 7415-7424, 1997.

34. Kim JY, Kim N, Yenari MA and Chang W: Hypothermia and pharmacological regimens that prevent overexpression and overactivity of the extracellular calcium-sensing receptor protect neurons against traumatic brain injury. J Neurotrauma 30: 1170-1176, 2013.

35. Schoch KM, Evans HN, Brelsfoard JM, Madathil SK, Takano J, Saido TC and Saatman KE: Calpastatin overexpression limits calpain-mediated proteolysis and behavioral deficits following traumatic brain injury. Exp Neurol 236: 371-382, 2012.

36. Sullivan PG, Rabchevsky AG, Waldmeier PC and Springer JE: Mitochondrial permeability transition in CNS trauma: Cause or effect of neuronal cell death? J Neurosci Res 79: 231-239, 2005.

37. Alexi T, Borlongan CV, Faull R, Williams CE, Clark RG, Gluckman PD and Hughes PE: Neuroprotective strategies for basal ganglia degeneration: Parkinson's and Huntington's diseases. Prog Neurobiol 60: 409-470, 2000.

38. Bar-Or D, Bar-Or R, Rael LT and Brody EN: Oxidative stress in severe acute illness. Redox Biol 4: 340-345, 2015.

39. Ehsaei M, Khajavi M, Arjmand MH, Abuee MA, Ghayour-Mobarhan $M$ and Hamidi Alamdari D: Prooxidant-antioxidant balance in patients with traumatic brain injury. Acta Neurol Belg 115: 69-73, 2015.

40. Freire MA: Pathophysiology of neurodegeneration following traumatic brain injury. West Indian Med J 61: 751-755, 2012.

41. Xue T, Luo P, Zhu H, Zhao Y, Wu H, Gai R, Wu Y, Yang B, Yang $X$ and He Q: Oxidative stress is involved in Dasatinib-induced apoptosis in rat primary hepatocytes. Toxicol Appl Pharmacol 261: 280-291, 2012.

42. Lau WK, Mak JC, Chan KH and Law AC: Cigarette smoke-induced cerebral cortical interleukin-6 elevation is not mediated through oxidative stress. Neurotox Res 22: 170-176, 2012.

43. Zelko IN, Mariani TJ and Folz RJ: Superoxide dismutase multigene family: A comparison of the CuZn-SOD (SOD1), Mn-SOD (SOD2), and EC-SOD (SOD3) gene structures, evolution, and expression. Free Radic Biol Med 33: 337-349, 2002.

44. Chelikani P, Fita I and Loewen PC: Diversity of structures and properties among catalases. Cell Mol Life Sci 61: 192-208, 2004.

45. Ansari MA, Roberts KN and Scheff SW: Oxidative stress and modification of synaptic proteins in hippocampus after traumatic brain injury. Free Radic Biol Med 45: 443-452, 2008.

46. Wang D, Yuan X, Liu T, Liu L, Hu Y, Wang Z and Zheng Q: Neuroprotective activity of lavender oil on transient focal cerebral ischemia in mice. Molecules 17: 9803-9817, 2012. 